\title{
Power Saving Routing Protocol for Ad hoc Networks based on AODV
}

\author{
Radha Rani Gupta \\ Department of Information \\ Technology, LNCT, Bhopal \\ M.P., India
}

\author{
Mahendra Ku. Mishra \\ Department of Information \\ Technology, LNCT, Bhopal \\ M.P., India
}

\author{
Manish Shrivastava \\ Department of Information \\ Technology, LNCT, Bhopal \\ M.P., India
}

\begin{abstract}
An ad hoc network is a wireless and infrastructure less network. It has dynamic and impermanent network topology. Each of nodes contains limited power in ad hoc networks. Power constraint is a major challenge to ad hoc networks. Limited power of nodes breaks link of the networks and reduces life of the networks. This proposed method minimizes the problem of link breakage and reduces the energy use of the network using a threshold (cutoff) based technique. This method takes all the paths whose nodes have energy greater than threshold value. This method selects the shortest path from the various paths based on AODV (Ad hoc on demand Distance Vector) protocol for routing. This proposed method is an extension of AODV protocol on the basis of energy factor and it shows better performance than existing AODV protocol. The performance parameters are throughput, packet delivery fraction and normalized routing load. It reduces network overhead and increases network lifetime. We use NS2 network simulator for performance analysis.
\end{abstract}

\section{General Terms}

Power, Performance analysis, power aware routing.

\section{Keywords}

Ad hoc Networks, Energy efficient routing, AODV, Network lifetime

\section{INTRODUCTION}

An ad hoc Network is a flexible network that can be setup anytime anywhere without any support of perquisite infrastructure. Due to its quick deployment and infrastructure less nature these networks have applications in areas such as defense and disaster areas. A routing protocol is used to have multi-hop communication in these networks. The main goal of a routing protocol is to send the data between a pair of nodes in timely. The routing protocol has several constraints such as dynamic, constantly changing topology, transmission power bandwidth and processing capability of the nodes [1].

In mobile ad hoc networks (MANET), every node works as a router and a terminal that can be a source or a destination. If operation of nodes fails then this degrades performance of network. For energy efficient routing it becomes necessary to use energy of node in efficient way because node has limited energy [2]. Energy efficiency plays an important role in ad hoc networks. Network life is reduced by using power in inefficient way. Energy aware routing protocols are designed for increasing the network lifetime and reducing energy consumption for data transferring through node to node in route searching process [3].We use a new method for increasing network lifetime and minimizing energy consumption that is based on AODV protocol. It is enhanced AODV that provide energy efficiency routing. The paper is summarized as section 2 and section 3 presents related work on energy efficient AODV protocol and brief description of AODV protocol respectively. In section 4 we present our proposed work that is enhanced AODV. In section 5 simulation and performance evaluation of our work is described. Section 6 concludes our proposed work.

\section{RELATED WORK}

There are numerous routing protocols for reducing energy consumption and increasing network lifetime that have been proposed. The REAQ (Route Stability and Energy Aware Quality of service) model provides reliable route discovery for both stability and residual energy metrics. It provides QoS (Quality of Service) routing in ad hoc networks. The proposed routing model added three fields in route request packet that are accumulated path stability, accumulated energy cost, minimum bandwidth and maximum delay and one field in route reply packet that is reliability factor. This model significantly increases the network lifetime. It decreases the QoS violation and problem of route breakage, by selecting reliable path for data transferring in the network [3]. The paper [4] shows that when mobility and number of nodes increases, AODV gives better performance than DSR protocol. It provides high performance in presence of noise and reduces packet dropping in the network. The proposed method gives high throughput, less delay, less overhead and consumes less energy. A power aware ad hoc routing protocol based on AODV is introduced that saves energy of the network. It includes a parameter that estimates mean power of entire network. In route searching process, intermediate nodes calculate the current mean power of the network and compare remaining energy with mean power [5].There is an efficient route searching algorithm and it includes two parameters one is total energy of path and other is residual battery power of the network. These parameters are added in every node of ad hoc networks. It uses AODV protocol and increases network lifetime [6]. In [2], authors proposed a threshold based algorithm using AODV. In this algorithm, any node can make a route if its energy is larger than threshold value. All the nodes energy is compared with threshold and these nodes are participated for making routes. This algorithm provides energy saving route and increases life of node in the network.

Ad hoc Quality of service on demand Routing (AQOR) protocol is a combination of on demand route searching and signaling functions for resource reservation of network. It provides quality of service violation detection and high performance of network [7]. This paper [1] proposes a routing strategy that takes this aspect into consideration by modifying the Route Request (RREQ) packet of the AODV routing protocol. The protocol chooses a threshold below which a node is not permitted to relay data/control packets. This paper [8] proposed a method that is Energy Efficiency and Secure Communication Protocol (EESCP). It uses two algorithms cluster election and diffie Hellman key exchange algorithm that divides network into 2-hop clusters. Nodes elect a cluster head that has maximum energy. 


\section{AD HOC ON DEMAND DISTANCE VECTOR (AODV)}

AODV is an on demand (reactive) ad hoc routing protocol. It supports both unicast and multicast routing. It maintains routing information when routing is required. In mobile ad hoc networks, each node stores routing information about neighboring nodes into routing table. AODV is a combination of DSDV and DSR ad hoc routing protocols. As DSR is a reactive protocol, $\mathrm{AODV}$ is also a reactive routing protocol and as DSDV uses sequence number AODV also uses sequence number that ensures freshness of route. When a fixed time is over routing information is expired that is route entry is deleted from routing table. AODV performs route searching and route maintenance operations [9].

In route searching operation, AODV uses two packets route request (RREQ) and route reply (RREP) packets. When source wish to communicate with destination and there is no direct route between them then source broadcasts RREQ packets to its neighbor nodes. RREQ packet contains destination address, source address, broadcast ID as a identifier, recent destination sequence number and source sequence number fields. When intermediate nodes receive RREQ packet then they reply by RREP packet to other intermediate nodes and forward to source. When destination receives RREQ, it unicast RREP through a shortest route to source and data is transferred from source to destination on that route. Fig 1 shows the route establishing process in AODV routing protocol.

As link is broken, a route maintenance operation is required. Node informs to its neighbor nodes by a route error RERR packet. This packet is forwarded to destination and back warded to source. Source initiates again route searching operation on receiving RERR packet to make a new route for transferring remaining data to destination.

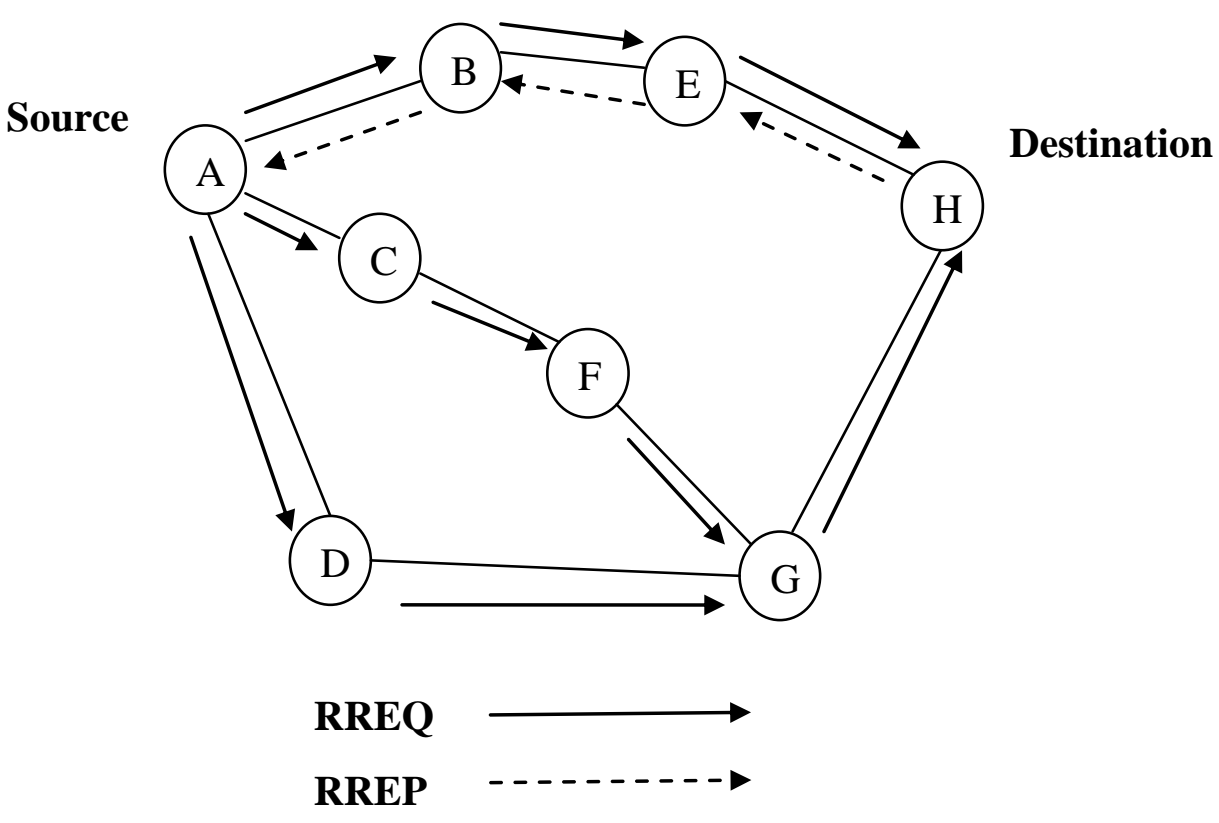

Fig 1: Route Establishment in AODV

\section{PROPOSED WORK}

\subsection{Problem Statement}

An ad hoc Network consists of spatially distributed autonomous system to communicate each other using intermediate nodes. All the mobile nodes are capable to handle route broadcast mechanism without need of centralize controller but one major challenge is, how to reduce power consumption of each mobile node's because each of node contains limited battery power. So this work encourages us to decide the field of saving energy consumption with efficient way. For that scheme, we apply threshold (cutoff) based technique and utilize minimum sleep power consumption under NS-2.31 simulation tool. We analyze the behavior of the network on the basis of throughput, normalized routing load, energy consumption of nodes and saving graph.

\subsection{Proposed Method}

In our proposal we reduce the energy consumption of the network using "Efficient AODV on the Bases Cutoff Power Saving Scheme under MANET" for that purpose very first we find out how the energy can be utilize by the mobile node here we identify some of energy discharge parameter like:
(a) Transmission Power
(b) Receiving Power
(c) Idle Power
(d) Sensing Power
(e) Sleep [Power Consumption in sleeping mode]

In above parameter base we utilize the mobile node energy and we get maximum power needed through if we data transmit and data receive case means transmission power and receiving power and minimum energy utilize if we apply ideal, sensing as well as sleep power consumption scheme, so our threshold (cut-off) base scheme provides minimum energy consumption as compare to existing scheme, in that scheme if node energy is less than the 10 joule then node goes into the sleeping state and energy consumption is lower because sleep case nearly ten time lower energy require as compare to 
transmission as well as receiving, and sender search the new route before all pervious intermediate node died that scheme provide more reliable as compare to previous schemes, in our proposal if node work under threshold condition so that time node require minimum power as compare to transmission and receiving energy and also switch new route if route search are completed.

\subsection{Threshold (cut-off) Based Scheme}

In threshold based scheme fix threshold value is used for path selection. Means the nodes energy value is compared with the threshold value; if it is greater than the threshold value then that node will be taken in to consideration otherwise it is discarded at the time of route search. And finally all the path whose nodes have energy value greater than the threshold value are taken in to consideration and the shortest path from them is selected based on AODV algorithm which is the best path based on threshold value scheme after the route selection if any node energy goes under threshold condition so we send data but sleep power utilization base that technique reduce the power consumption.

\subsection{Example}

In fig 2, node $\mathrm{A}$ is a source and $\mathrm{E}$ is a destination. There is no direct route from node $\mathrm{A}$ to node $\mathrm{E}$, so source A broadcasts route request (RREQ) packet to its neighboring nodes. The intermediate nodes are "B","C","D" and "F" between A and E. In route searching process, first energy of node is compared with threshold value that is 10 joule. There are three routes from source to destination but node B has 9 joule energy that is less than threshold value so it is not taken into path selection. Other two routes $\mathrm{A}-\mathrm{C}-\mathrm{E}$ and $\mathrm{A}-\mathrm{D}-\mathrm{F}-\mathrm{E}$ are selected for routing, path $\mathrm{A}-\mathrm{C}-\mathrm{E}$ is shortest path so it is selected for data transmission. Hence route reply (RREP) packet is sent from $\mathrm{E}$ to $\mathrm{C}$ and $\mathrm{C}$ to $\mathrm{A}$. Source node $\mathrm{A}$ receives RREP packet and starts data transferring on the route $\mathrm{A}-\mathrm{C}-$ E.

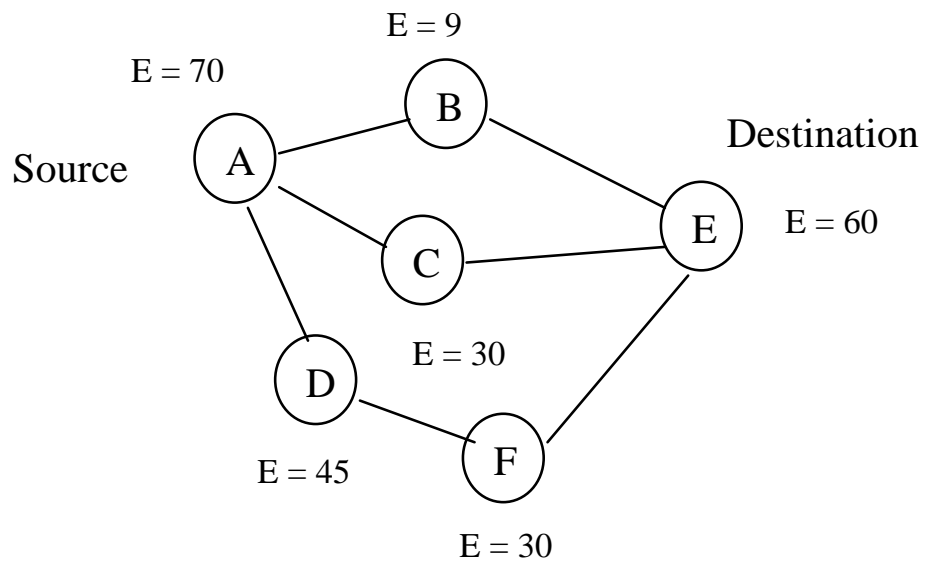

Fig 2: Route establishment in proposed method

\section{SIMULATION AND RESULT}

In this section, we analyze performance of our proposed method and it is compared with protocol AODV. In proposed method simulation, we take 50 nodes that have random energy. Simulation is done in NS2.31 network simulator and traffic model is generated by EAODV.tcl file. In this method we use two traffic types Constant Bit Rate (CBR) and File Transfer Protocol (FTP).

\subsection{Performance Metrics}

(i) Energy consumption (EC): The amount of Energy spent by node to transmit number of Data packets and is given below

$\mathbf{E C}=$ Initial Energy assigned to node - The energy used to send data packet

(ii) Average Energy Consumption (AEC): It is an average energy consumed by an each node to transmit data packets from source to destination by multi hop routing is given below

AEC $=\frac{\text { ECbynodesinof nodesinmultihop }}{\text { Numberofnodesinmultihop }}$

(iii) Packet Delivery Fraction (PDF): The ratio of total numbers of data packets successfully delivered to the total number of data packets sent and is given below
PDF $=\frac{\text { Datapacketssuccessfullydelivered }}{\text { Numberof packetssent }} * 100 \%$

(iv) Throughput (T): The ratio of number of packets delivered to the destination to the time required to travel from source to destination is normally represented by Bits per Second (bps) and is given below

$\mathbf{T}=\frac{\text { Number of packets deliverd }}{\text { Time required to travel from source to destination }}$

(v) End-to-End Delay (EED): It is the delay between source to destination nodes. It is measured by summation of delays of number of hops involved between source to destination using given equation

$\mathbf{E E D}=\sum_{\mathbf{i}=\mathbf{1}}^{\mathbf{n}} \mathbf{d i} \quad$ where $\mathrm{d}$ is hop delay

(vi) Normalized Routing Load (NRL): It is the ratio of number of routing packets and number of received packets at the destination.

NRL $=\frac{\text { Numberof routingpackets }}{\text { Numberof receivingpackets }}$

\subsection{Simulation Parameters}

We have simulated it in NS2.31. Simulation parameters are listed in the table below. 
Table 1. Simulation parameters

\begin{tabular}{|c|c|}
\hline Parameter Name & Value \\
\hline Network Topology & $800 \mathrm{~m} \times$ 800m \\
\hline Number of Nodes & 50 \\
\hline Mobility Model & Random Way Point \\
\hline Traffic Type & CBR and FTP \\
\hline Simulation Time & $100 \mathrm{sec}$ \\
\hline Routing Protocol & AODV \\
\hline
\end{tabular}

\begin{tabular}{|c|c|}
\hline Packet Size & 512 byte \\
\hline Transmission Range & $550 \mathrm{~m}$ \\
\hline Node Mobility & Random \\
\hline txPower & $1.5 \mathrm{~W}$ \\
\hline rxPower & $1.0 \mathrm{~W}$ \\
\hline Sleep Power & $0.1 \mathrm{~W}$ \\
\hline
\end{tabular}

\subsection{Performance Analysis}

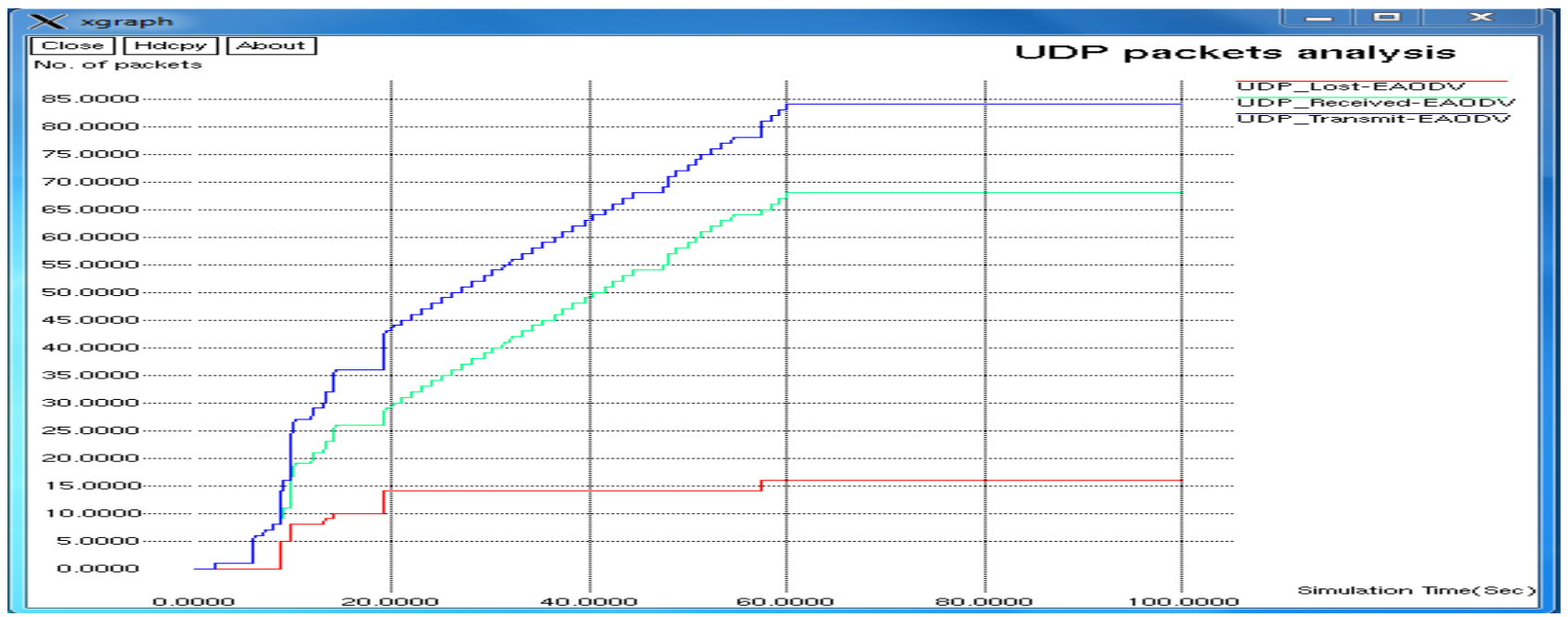

Fig 3: UDP Packet analysis in AODV

The proposed power aware ad hoc network routing protocol is implemented in NS 2.31. Packet delivery fraction, normalized routing load and throughput parameter have been compared with AODV protocol. In NS-2.31, xgraphs represent the graphical view of results. Fig 4 shows that the udp packet delivery rate increases and udp packet drop rate decreases in our proposed method as compared to AODV which is mentioned in fig 3 under the same environment. Proposed method consumes less energy in sleep mode that saves unnecessary wastage of node's energy in ad hoc networks and it provides longer network lifetime than AODV protocol.

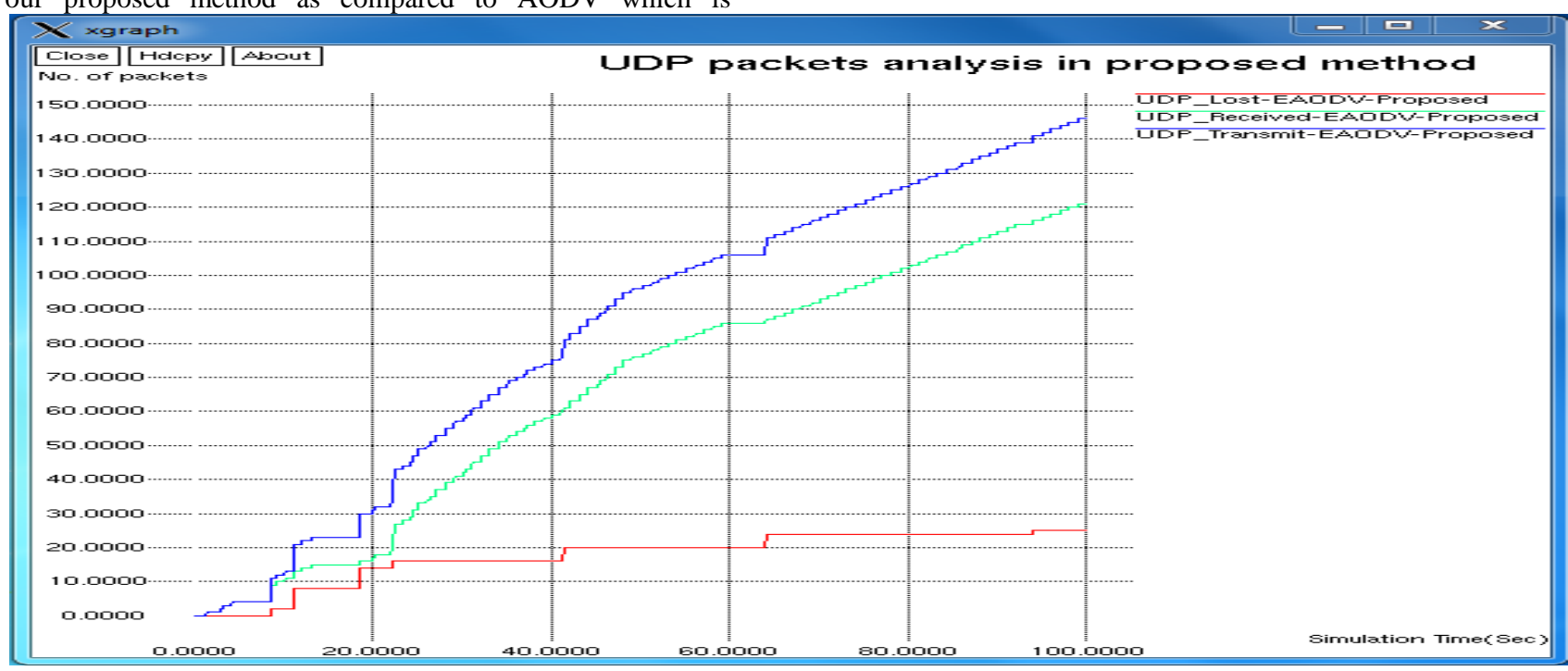

Fig 4: UDP Packet analysis in Proposed Method 
Fig 5 is a comparison xgraph of routing load and shows that normalized routing load increases with increases simulation time in both protocols AODV and our proposed modified
AODV protocol. From fig 5 it is clear shown that our proposed method reduces routing load $22 \%$ as compared to existing AODV protocol.

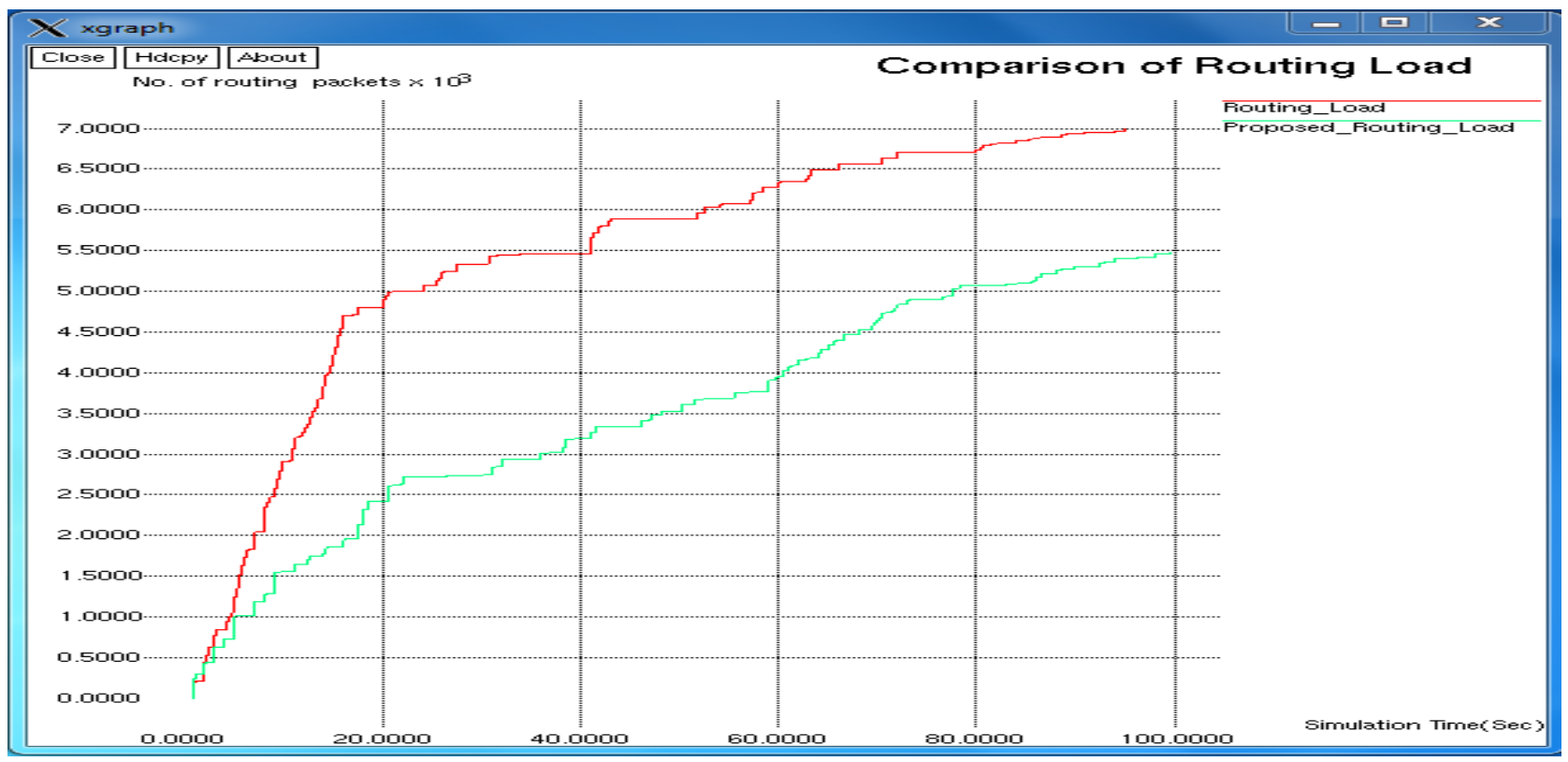

Fig 5: Normalized Routing Load

Fig 6 is a comparison xgraph of throughput between AODV and proposed protocol that shows our proposed protocol provides high throughput.

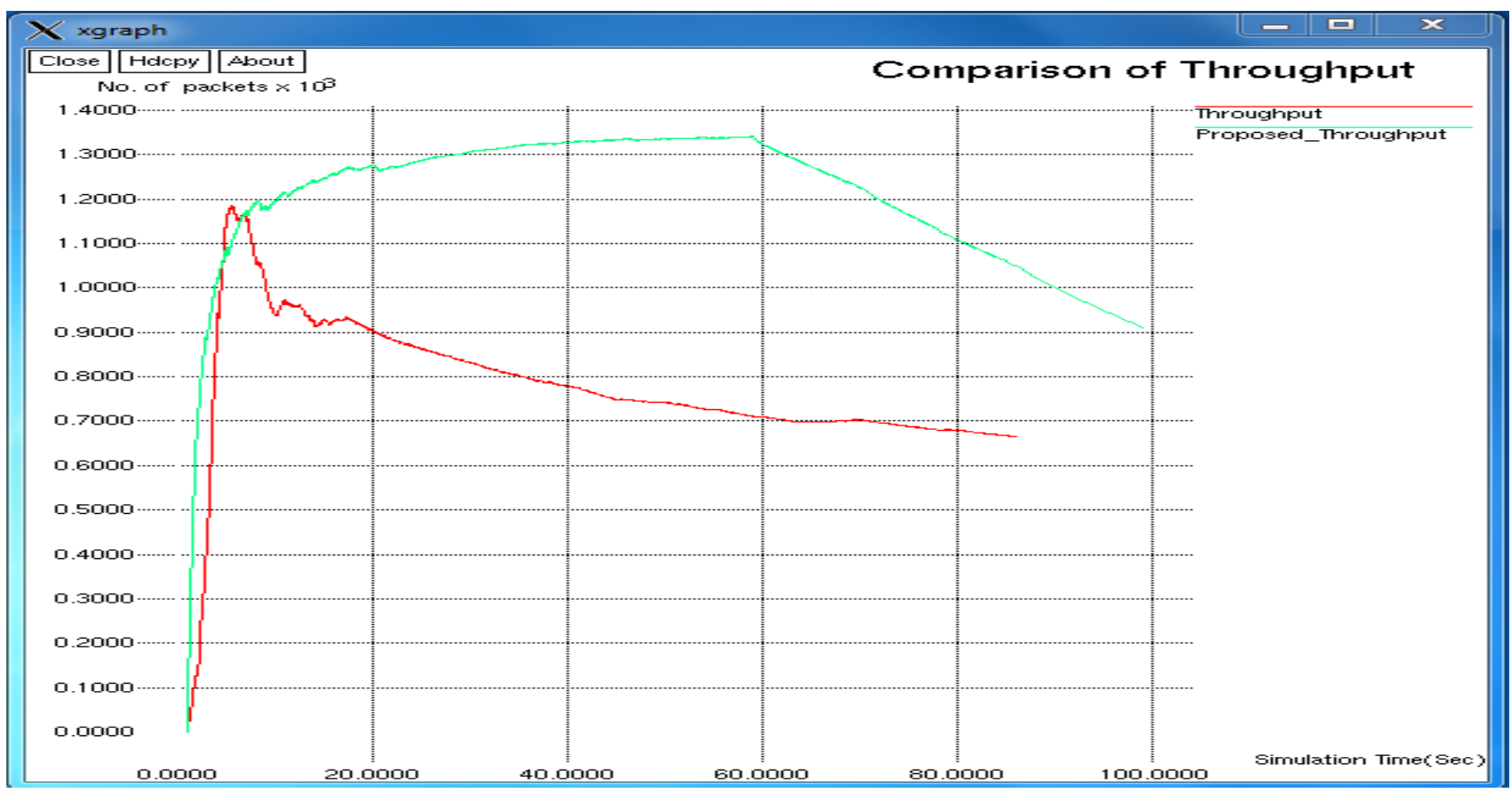

Fig 6: Throughput

\section{CONCLUSION}

This paper presents an energy aware routing protocol based on AODV. In our proposed method, energy of node is compared with threshold value. If node energy is greater than threshold then node is selected for routing. All nodes are selected to make route that have greater value than threshold.
Result of proposed method is better than existing AODV protocol. Packet delivery fraction, throughput and normalized routing load are performance parameters. It reduces energy consumption, increases network lifetime and provide high performance. This proposed method can be simulated in different routing protocol with security concept. 


\section{ACKNOWLEDGMENTS}

The authors would like to express their thank to the anonymous reviewers for their valuable suggestions.

\section{REFERENCES}

[1] Prem Chand and M.K.Soni," A Novel Routing Scheme for Mobile Ad Hoc Network", I. J. Computer Network and Information Security, 4, pp. 17-24, 2013

[2] TriptiNema, AkhileshWaoo, P.S.Patheja and Sanjay Sharma, "Energy Efficient Adaptive Routing Algorithm in MANET with Sleep Mode", International Journal of Advanced Computer Research, Volume-2 Number-4 Issue-6 December-2012.

[3] P.Srinivasan and Dr. P. Kamalakkannan, "REAQAODV: Route Stability and Energy Aware QoS Routing in Mobile Ad hoc Networks ", IEEE- Fourth International Conference on Advanced Computing, ICoAC 2012, MIT, Anna University, Chennai. December 13-15, 2012.

[4] G.Rajkumar, R.Kasiram and D.Parthiban, "Optimized QoS Metrics and Performance Comparison of DSR and AODV Routing Protocols",IEEE-International Conference On Advances In Engineering, Science And Management (ICAESM -2012) March 30, 31, 2012.

[5] Rajesh kumarYadav and Deeptkpanwar," Optimal and Robust Framework for Enhancing Network Lifetime using Power Efficient AODV in Mobile Ad hoc Network", IEEE-201SO ICCCNT12, Coimbatore, India ,26th -28th July 2012.

[6] Seema Verma, Pinki Nayak and Rekha Agarwal, "Energy Efficient Routing in Mobile Adhoc Networks based on AODV Protocol", IJCSI International Journal of Computer Science Issues, Vol. 9, Issue 6, No 2, pp. 344-349, November 2012.

[7] Qi Xue and Aura Ganz," Ad hoc QoS on-demand routing (AQOR) in mobile ad hoc networks", Journal Parallel Distributed Computing,63 , pp. 154-165, 2003.

[8] G. Siva Kumar, M. Kaliappan and L. JerartJulus," Enhancing the Performance of MANET using EESCP", IEEE Proceedings of the International Conference on Pattern Recognition, Informatics and Medical Engineering, March 21-23, 2012.

[9] CharuWahi and Sanjay Kumar Sonbhadra, "Mobile Ad Hoc Network Routing Protocols: A Comparative Study", International Journal of Ad hoc, Sensor \& Ubiquitous Computing (IJASUC) Vol.3, No.2, April 2012

[10] C. Siva Ram Murthy and B. S. Manoj, "Ad hoc Wireless Networks Architectures and Protocols", Pearson Education, Prentice Hall, 2004. 